\title{
Parametric Analysis of Tubular-Type Linear Magnetic Couplings with Halbach Array Magnetized Permanent Magnet by Using Analytical Force Calculation
}

\author{
Chang-Woo Kim and Jang-Young Choi* \\ Department of Electrical Engineering, Chungnam National University, Daejeon 34134, Korea
}

(Received 1 December 2015, Received in final form 1 February 2016, Accepted 11 February 2016)

\begin{abstract}
Magnetic couplings are widely used in various industrial applications because they can transmit magnetic force without any mechanical contact. In addition, linear couplings have many advantages. For example, they do not need to convert rotary motion to linear motion. This paper shows an analytical analysis of tubular type linear magnetic couplings (TLMCs) with a Halbach array magnetized permanent magnet (PM). An analytical method for magnetic fields owing to PMs is performed by using magnetic vector potential as well as Poisson and Laplace equations. Then, the magnetic force is calculated by using the Maxwell stress tensor. The analytical analysis results were compared with finite element method (FEM) results. In addition, we predicted the magnetic force characteristic according to design parameters such as the iron core thickness, inner PM thickness to -outer PM thickness ratio, PM segment ratio of the axial magnetized PM segment and radial magnetized PM segment, and various pole numbers.
\end{abstract}

Keywords : Parametric analysis, Halbach array, magnetic coupling, analytical method

\section{Introduction}

Linear machines must consider end effects and have low efficiency. However, they do not need to convert rotary motion to linear motion. Linear machines have higher dynamic performance, improved reliability, and a reduced power loss. In addition tubular linear machines are very attractive compared with flat linear machines, because the former produce high magnetic force owing to a high permanent magnet (PM) coefficient of utilization and do not have end turn effects [1-3].

Tubular-type linear magnetic couplings (TLMCs) are used to transmit the magnetic force from actuators to loads without any mechanical contact. Analyzing TLMCs with a Halbach array magnetized PM magnetic force is very important because this force acts as an overload protection in the machines. Therefore this paper predicts the magnetic force characteristics according to design parameters such as iron core thickness, inner PM thickness to -outer PM thickness ratio, PM segment ratio, and pole numbers. To acquire magnetic force analysis results of TLMCs, we used an analytical force calculation. An

(C)The Korean Magnetics Society. All rights reserved.

*Corresponding author: Tel: +82-42-821-7610

Fax: +82-42-822-4933, e-mail: choi_jy@cnu.ac.kr analytical method for magnetic fields is performed by using magnetic vector potential as well as Poisson and Laplace equations [4-6]. Then we calculated the magnetic force using the Maxwell stress tensor [7]. The analysis results were compared with finite element method (FEM) results.

\section{Analysis of TLMC}

Figure 1 shows the concept of the TLMC, which consists of the iron and PMs. It transmits the magnetic force from actuators to loads without any mechanical contact.

\subsection{Analytical Model}

Figure 2 shows a schematic of the TLMC that is composed of cylindrical coordinate systems. The TLMC consists of an outer iron core, outer PMs, air gap, inner PMs, and inner iron core. For the convenience of the analytical solutions, the permeability of the iron cores is assumed to be infinite, and the permeability of the PMs is assumed to have the permeability of air.

The Halbach array magnetization model of the outer PMs, and inner PMs for a Fourier series expansion is shown in Fig. 3 (a) and Fig. 3 (b), respectively. The magnetization can be expressed as 


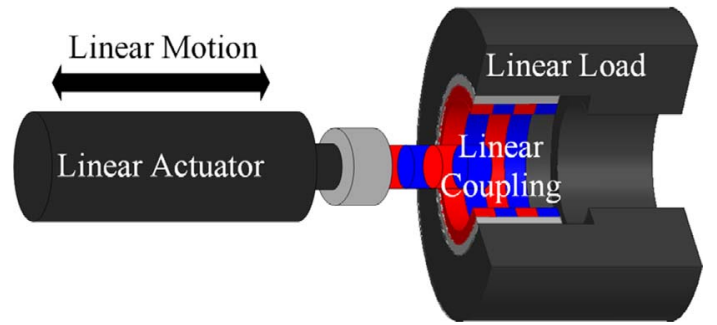

Fig. 1. (Color online) Concept of the TLMC.

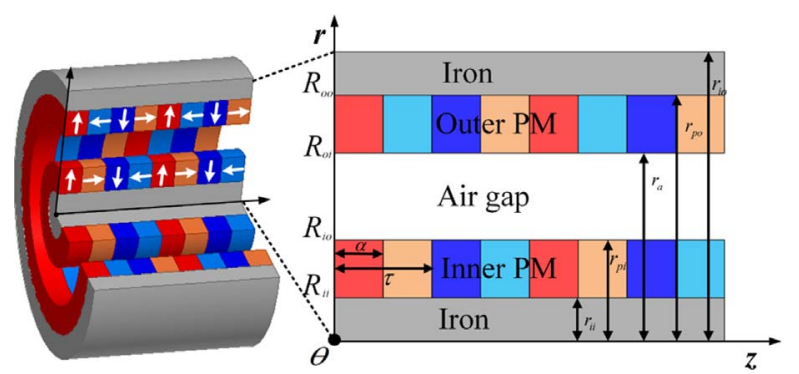

Fig. 2. (Color online) Schematic of the TLMC with Halbach array magnetized PM.
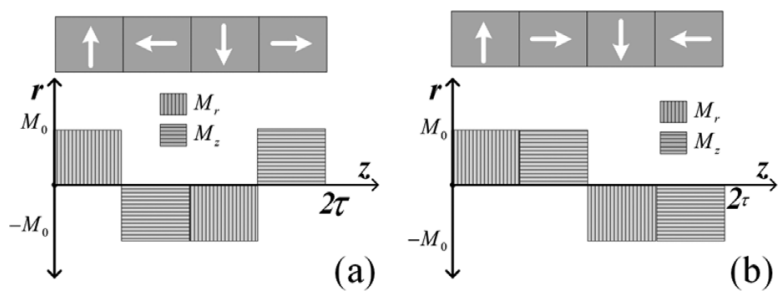

Fig. 3. Halbach array magnetization: - (a) Outer PM, (b) Inner PM.

$$
\begin{aligned}
& \mathbf{M}_{i}=\sum_{n=-\infty}^{\infty} M_{r n} e^{-j k_{n} z} i_{r}+\sum_{n=-\infty}^{\infty} M_{z n} e^{-j k_{n} z} i_{z} \\
& \mathbf{M}_{o}=\sum_{n=-\infty}^{\infty} M_{r n} e^{-j k_{n} z} i_{r}-\sum_{n=-\infty}^{\infty} M_{z n} e^{-j k_{n} z} i_{z} \quad k_{n}=\frac{n \pi}{\tau}
\end{aligned}
$$

where $i_{r}$ is the radial unit vector, $i_{z}$ is the axial unit vector, and $k_{n}$ is the $\mathrm{n}^{\text {th }}$-order space harmonic.

\subsection{Magnetic_Field_Produced PMs}

In the analytical model, by using flux density B, and magnetic intensity $\mathrm{H}$, the governing equations are induced from the following equation :

$$
\mathbf{B}=\mu_{0}(\mathbf{H}+\mathbf{M})
$$

Applying the curl operator to both sides of (3), Laplace equations and Coulomb's gauge lead to the governing equations in each region:

$$
\begin{aligned}
& \nabla^{2} \mathbf{A}^{\mathrm{I}}=-\mu_{0}\left(\nabla \times \mathbf{M}_{i}\right) \\
& \nabla^{2} \mathbf{A}^{\mathrm{II}}=0 \\
& \nabla^{2} \mathbf{A}^{\mathrm{III}}=-\mu_{0}\left(\nabla \times \mathbf{M}_{o}\right)
\end{aligned}
$$

The superscripts I, II, and III denote the outer PM regions, air gap, and inner PM regions, respectively. The magnetic vector potential, A, can be written as

$$
\mathbf{A}=\sum_{n=-\infty}^{\infty} A_{n}(r) e^{-\left(j k_{n} z+m_{k} \theta\right)} i_{\theta}
$$

Using (3) and (4), we can derive the magnetic vector potential as it relates to the magnetizations. By substituting (4) into (5), the magnetic vector potential $\mathrm{A}$ is expressed as

$$
\begin{aligned}
& A_{n}^{I}(r)=C_{n}^{I} I_{1}\left(k_{n} r\right)+D_{n}^{I} K_{1}\left(k_{n} r\right)-\frac{j \mu_{0} \mathbf{M}_{\mathbf{r n} \_\mathbf{o}}}{k_{n}} \\
& A_{n}^{I I}(r)=C_{n}^{I I} I_{1}\left(k_{n} r\right)+D_{n}^{I I} K_{1}\left(k_{n} r\right) \\
& A_{n}^{I I I}(r)=C_{n}^{I I I} I_{1}\left(k_{n} r\right)+D_{n}^{I I I} K_{1}\left(k_{n} r\right)-\frac{j \mu_{0} \mathbf{M}_{\mathbf{r n} \_\mathbf{i}}}{k_{n}}
\end{aligned}
$$

where $I_{1}$ and $K_{1}$ are the modified Bessel functions of the first and second kind of order 1, respectively.

Finally, the radial magnetic flux density (8) and axial magnetic flux density (9) are derived using the definition of the magnetic vector potential.

$$
\begin{aligned}
& \nabla \times \mathbf{A}=\mathbf{B} \\
& \mathbf{B}_{\mathbf{r}}=-\frac{\partial \mathbf{A}}{\partial z}=j k_{n} \mathbf{A} e^{-j k_{n} z} \\
& \mathbf{B}_{\mathbf{z}}=\left(\frac{\mathbf{A}}{r}+\frac{\partial \mathbf{A}}{\partial r}\right) e^{-j k_{n} z}
\end{aligned}
$$

\begin{tabular}{|c|c|c|}
\hline Boundary & Normal component & Tangential component \\
\hline $\mathbf{R}_{\mathrm{oo}}$ & & $\mathbf{B}_{\mathbf{z}}^{\mathbf{I}}=\mu_{0} \mu_{r} \mathbf{M}_{\mathbf{z n}} e^{-j k_{n} z}$ \\
\hline $\mathbf{R}_{\mathrm{oi}}$ & $\mathbf{B}_{r}^{\mathrm{I}}=\mathbf{B}_{\mathrm{r}}^{\mathrm{II}}$ & $\mathbf{B}_{\mathbf{z}}^{\mathbf{I}}=\mathbf{B}_{\mathbf{z}}^{\mathbf{I I}}+\mu_{0} \mu_{r} \mathbf{M}_{\mathbf{z n}} e^{-j k_{n} z}$ \\
\hline $\mathbf{R}_{\mathrm{io}}$ & $\mathbf{B}_{r}^{I I}=\mathbf{B}_{r}^{I I I}$ & $\mathbf{B}_{\mathbf{z}}^{\mathrm{II}}=\mathbf{B}_{\mathbf{z}}^{\mathrm{III}}-\mu_{0} \mu_{r} \mathbf{M}_{\mathbf{z n}} e^{-j k_{n} z}$ \\
\hline $\mathbf{R}_{\mathrm{ii}}$ & & $\mathbf{B}_{\mathbf{z}}^{\mathrm{III}}=\mu_{0} \mu_{r} \mathbf{M}_{\mathbf{z n}} e^{-j k_{n} z}$ \\
\hline
\end{tabular}

The undefined coefficients $C_{n}$ and, $D_{n}$ are determined using the boundary conditions reported in Table 1 .

\subsection{Magnetic Force}

In this analysis model, the inner iron core and PMs move in the axial direction. The interaction between the inner and outer PMs occurs a magnetic force, F. The magnetic force, $\mathbf{F}$, can be derived using the Maxwell stress tensor[8].

$$
\begin{aligned}
& \mathbf{F}_{\mathbf{z}}=\frac{1}{\mu_{0}} \int_{s} \mathbf{B}_{\mathbf{z}} \mathbf{B}_{\mathbf{r}} \\
& \mathbf{F}_{\mathbf{z}}=\frac{1}{\mu_{0}} \int_{0}^{l} \int_{0}^{2 \pi} \mathbf{B}_{\mathbf{z}} \mathbf{B}_{\mathbf{r}} r d \theta d z
\end{aligned}
$$

Table 1. Boundary condition at each boundary. 
Expanding (10) can express as

$$
\mathbf{F}_{\mathbf{z}}=-\frac{S}{2 \mu_{0}}\left\{\mathbf{B}_{\mathbf{r}}^{\mathrm{II}}\left(\mathbf{B}_{\mathbf{z}}^{\text {II }}\right)^{*}\right\}
$$

where the superscript $*$ signifies the complex conjugate, and the character, $S$, is the top surface of the inner PMs. The character, $S$, depends on the time. Thus, it can be expressed as

$$
S=2 \pi R_{o i}(l-\mathbf{v} t)
$$

where $l$ is the $\mathrm{z}$-axis directional length of the PMs, and $\mathbf{v}$ is the velocity of the moving core.

\subsection{Comparison of Analytical Calculations with FEM}

Prior to the parametric analysis, the accuracy of the analytical solution is confirmed using finite element methods (FEMs). We designed a TLMC with Halbach array magnetized PMs that have the initial parameter values listed in Table 2. Figure 4 shows a 3-D FEM

Table 2. Initial parameter values.

\begin{tabular}{cccc}
\hline \hline Parameter & Value & Parameter & Value \\
\hline z-directional length & $40 \mathrm{~mm}$ & Inner PM thickness & $6 \mathrm{~mm}$ \\
r-directional length & $30 \mathrm{~mm}$ & Outer PM thickness & $6 \mathrm{~mm}$ \\
Pole numbers & 4 & Iron thickness & $5 \mathrm{~mm}$ \\
\hline Magnet Volume & $45.2 \mathrm{~cm}^{3}$ & Pole segment ratio & $1: 1$
\end{tabular}

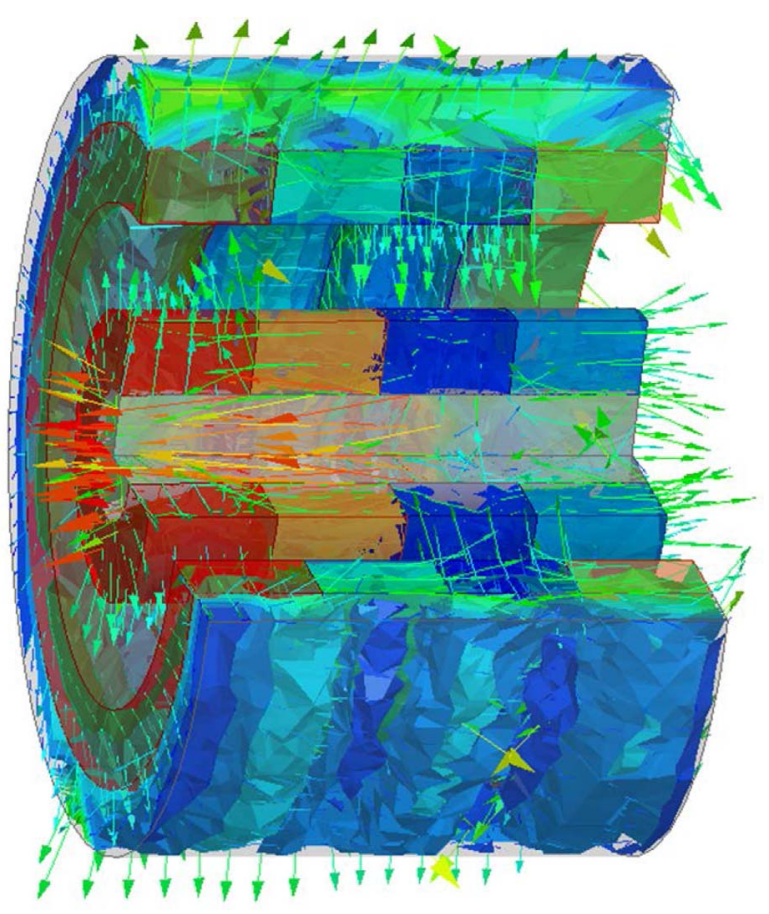

Fig. 4. (Color online) 3-D FEM analysis result of magneto static field distribution owing to PMs.

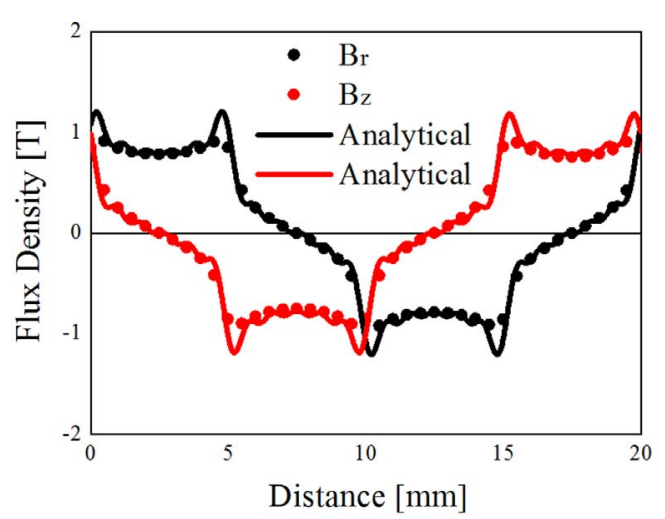

Fig. 5. (Color online) Comparison of analytical solution results with FEM results for flux density at surface of outer PMs.

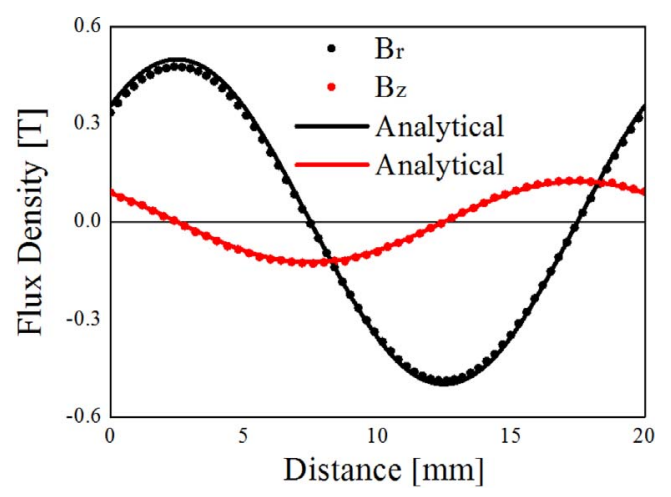

Fig. 6. (Color online) Comparison of analytical solution results with FEM results for flux density at airgap.

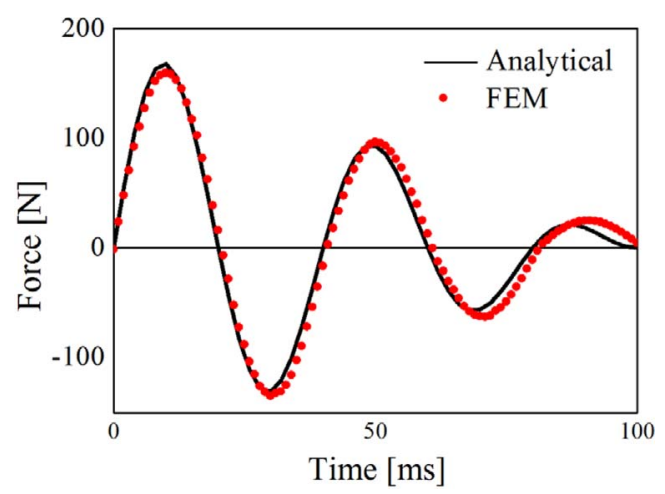

Fig. 7. (Color online) Comparison of analytical solution results with FEM results for magnetic force of initial model.

analysis result of magneto-static field distribution owing to PMs. Figure 5 and Fig. 6 show a comparison of analytical solution results with FEM results for the flux density at the surface of the outer PMs and air gap. Figure 7 shows a comparison of analytical solution results with FEM results for magnetic force. The error is caused by leakage flux and the end effect. Therefore, the analytical solution results are in good agreement with the FEM results. 


\section{Parametric Analysis}

In the initial design of the TLMC, an analytical solution is used to investigate the influence of the parameters on TLMC force. Here, force analysis is determined by the outer iron core thickness, inner PM thickness-outer PM thickness ratio, and PM segment ratio of the axial magnetized PM segment and radial magnetized PM segment.

\subsection{Force Analysis According to Outer Iron Core Thickness}

For a fixed value of length in the axial direction, and radial direction, Fig. 8 shows the variations of magnetic force with respect to the outer iron core thickness for 2 poles, 4 poles, 5 poles, and 8 poles. It can be observed for each number of poles that the magnetic force increases, as the outer iron core thickness decreases. However, considering the saturation of the iron core, $\mathrm{r}_{\mathrm{po}} / \mathrm{r}_{\mathrm{io}}=0.83$ is the most suitable ratio. And Fig. 9 shows the saturation effects at $\mathrm{r}_{\mathrm{po}} / \mathrm{r}_{\mathrm{io}}=0.87$ by using 2-D FEM.

\subsection{Force Analysis According to Inner PM Thickness- Outer PM Thickness Ratio}

For a fixed value of the air gap length and $r_{p o} / r_{i o}$ ratio, Fig. 10 shows the variations of magnetic force with respect to the inner PM thickness to -outer PM thickness

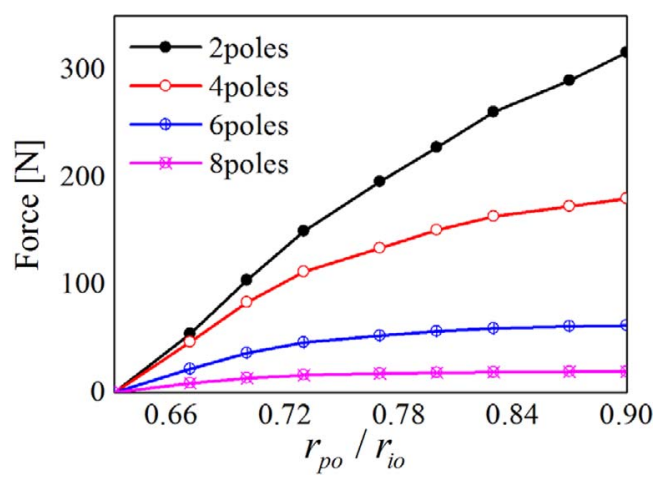

Fig. 8. (Color online) Variations of magnetic force according to the outer iron core thickness for various pole numbers.

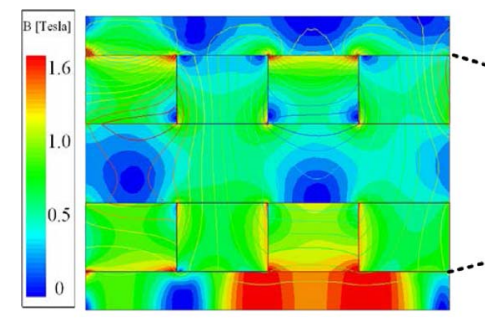

$\mathrm{r}_{\mathrm{po}} / \mathrm{r}_{\mathrm{io}}=0.87$

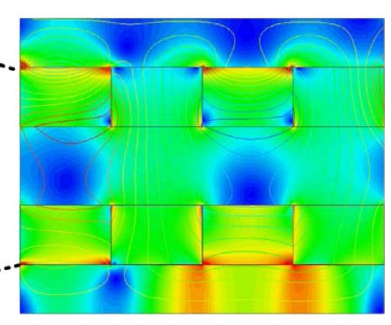

$\mathrm{r}_{\mathrm{po}} / \mathrm{r}_{\mathrm{io}}=0.83$
Fig. 9. (Color online) Saturation effect according to the outer iron core thickness by using 2-D FEM. ratio for various numbers of poles. It can be observed for each number of poles that the magnetic force is the highest, when the inner PM thickness-outer PM thickness ratio is 7:5. In Fig. 10, the amount of the PM increases as the outer PM thickness increases. However, magnetic force does not depend only on the amount of the PM, because of leakage from the air gap.

\subsection{Force Analysis according to PM Segment Ratio}

For a fixed value of PM thickness, Fig. 11 shows the variations of magnetic force with respect the radial magnetization PM segment-axial magnetization PM segment ratio for various numbers of poles. For 2 poles, it can be observed that the magnetic force is the highest, as radial magnetization PM segment-axial magnetization PM segment ratio is $7: 3$. When the poles are increased, it can be observed that the magnetic force is the highest when the radial magnetization PM segment-axial magnetization PM segment ratio is 5:5.

\subsection{Proposed Parameter Values}

For a fixed value of $\mathrm{z}$-directional length, r-directional

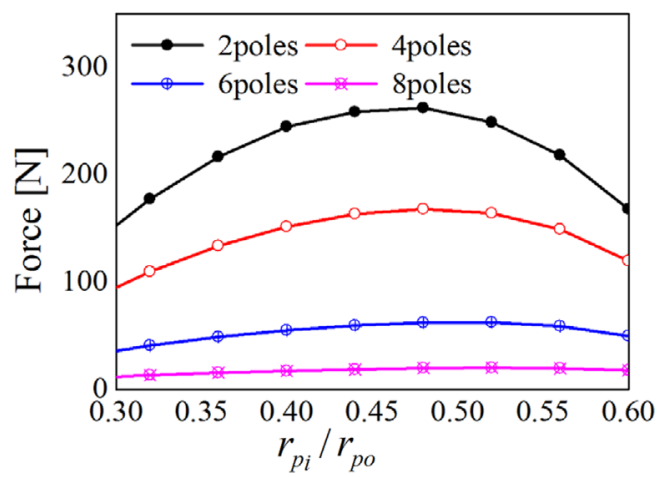

Fig. 10. (Color online) Variations of magnetic force according to inner PM thickness-outer PM thickness ratio for various pole numbers.

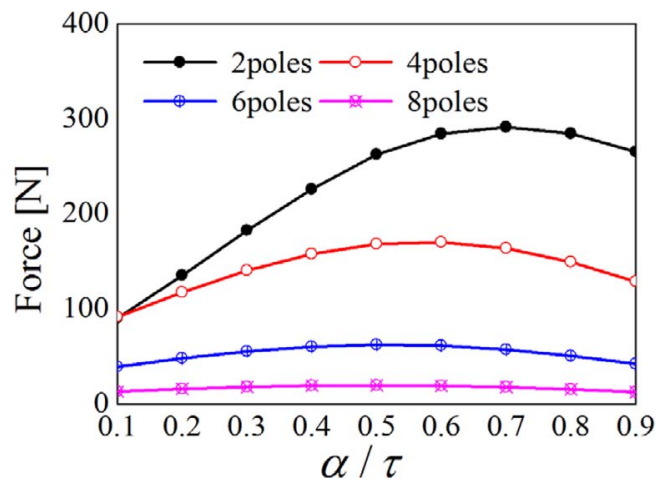

Fig. 11. (Color online) Variations of magnetic force according to the PM segment ratio for various pole numbers. 
Table 3. Proposed parameter values.

\begin{tabular}{cccc}
\hline \hline Parameter & Value & Parameter & Value \\
\hline z-directional length & $40 \mathrm{~mm}$ & Inner PM thickness & $7 \mathrm{~mm}$ \\
r-directional length & $30 \mathrm{~mm}$ & Outer PM thickness & $5 \mathrm{~mm}$ \\
Pole numbers & 2 & Iron thickness & $5 \mathrm{~mm}$ \\
Magnet Volume & $43.2 \mathrm{~cm}^{3}$ & Pole segment ratio & $7: 3$ \\
\hline
\end{tabular}
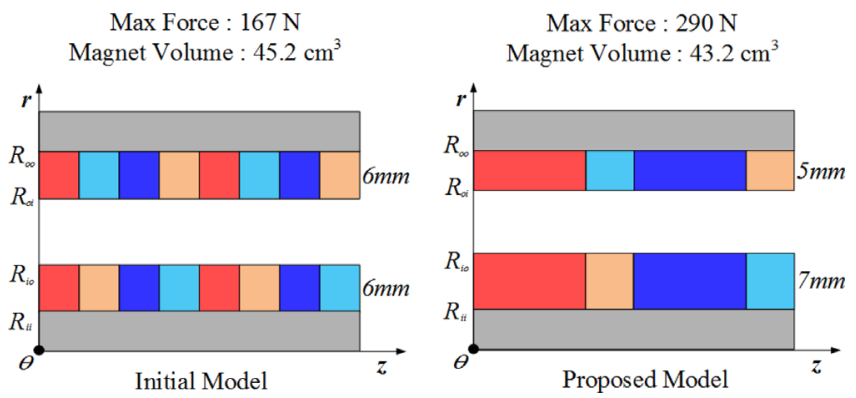

Fig. 12. (Color online) Schematic of the initial model and proposed model.

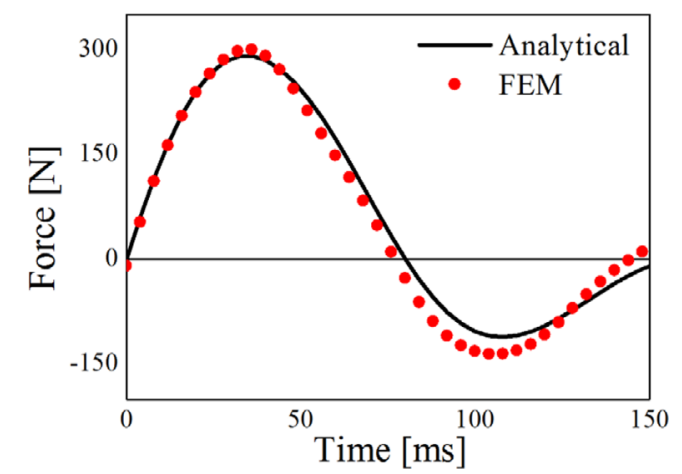

Fig. 13. (Color online) Comparison of analytical solution results with FEM results for magnetic force of proposed model.

length, and air- gap length, we predict the magnetic force based on various parameters. Finally, we derived the proper design parameters, which are listed in Table 3. Figure 12 shows a schematic of the proposed TLMC. Figure 13 shows a comparison of the analytical solution results with FEM results for the magnetic force of the proposed model.

\section{Conclusion}

This paper shows the magnetic field characteristics of a TLMC with Halbach array magnetization using an analytical method. In addition, the magnetic force was calculated using the Maxwell stress tensor. The analytical results are in good agreement with the FEM results. Finally, the influence of the iron core thickness, inner PM thickness-outer PM thickness ratio, and PM segment ratio design parameters on the performance of the TLMC were investigated using magnetic force calculations. The analytical method should be very useful in the initial design and optimization process of TLMCs with Halbach array magnetized PMs.

\section{Acknowledgements}

This work was supported by the Basic Science Research Program through the Ministry of Science, ICT and Future Planning, National Research Foundation of Korea, under Grant 2014R1A1A1A05002745.

\section{References}

[1] S. M. Jang, J. Y. Choi, H. W. Cho, and S. H. Lee, IEEE Trans. Magn. 41, 2028 (2005).

[2] W. Jiabin, W. Weiya, G. W. Jewell, and D. Howe, IEEE Trans. Ind. Magn. 49, 640 (2002).

[3] J. Wang, G. W. Jewell, and D. Howe, IEEE Trans. Magn. 35, 1986 (1999).

[4] H. B. Kang and J. Y. Choi, J Electr. Eng. Tech. Magn. 9, 926 (2014).

[5] R. Ravaud, G. Lemarquand, V. Lemaruand, and C. Depollier, IEEE Trans. Magn. 45, 1950 (2009).

[6] R. Ravaud, V. Lemarquand, and Guy Lemarquand, IEEE Trans. Magn. 46, 3860 (2010).

[7] David L. Trumper, W. J. Kim, and Mark E. Williams, IEEE Trans. IAS. 32, 371(1996).

[8] B. L. J. Gysen, Messen, K. J., Paulides, J. J. H., and Lomonova, IEEE Trans. Magn. 46, 39 (2010). 